\title{
Towards elucidation of zero-temperature criticality of the Ising model on 2D dynamical triangulations
}

\author{
Jan Ambjørn, ${ }^{1,2, *}$ Yuki Sato $\odot,{ }^{3,4, \dagger}$ and Tomo Tanaka ${ }^{5, \$}$ \\ ${ }^{1}$ The Niels Bohr Institute, Copenhagen University Blegdamsvej 17, DK-2100 Copenhagen, Denmark \\ ${ }^{2}$ IMPP, Radboud University, Heyendaalseweg 135, 6525 AJ Nijmegen, The Netherlands \\ ${ }^{3}$ Institute for Advanced Research, Nagoya University Chikusaku, Nagoya 464-8602, Japan \\ ${ }^{4}$ Department of Physics, Nagoya University Chikusaku, Nagoya 464-8602, Japan \\ ${ }^{5}$ Department of Physics, Waseda University Okubo, Tokyo 169-8555, Japan
}

(Received 27 March 2020; accepted 30 April 2020; published 18 May 2020)

\begin{abstract}
We study the zero-temperature criticality of the Ising model on two-dimensional dynamical triangulations to contemplate its physics. As it turns out, an inhomogeneous nature of the system yields an interesting phase diagram and the physics at the zero temperature is quite sensitive about how we cool down the system. We show the existence of a continuous parameter that characterizes the way we approach the zero-temperature critical point and it may enter in a critical exponent.
\end{abstract}

DOI: 10.1103/PhysRevD.101.106019

\section{INTRODUCTION}

The Ising model on two-dimensional (2D) dynamical triangulations (DT) is a statistical system including quantum effects of gravity, which was studied first by Boulatov and Kazakov [1,2]. ${ }^{1}$ We call this model the BoulatovKazakov Ising model. Through the use of matrix models, the Boulatov-Kazakov Ising model was solved exactly and all the critical exponents were obtained analytically [2]. Those are different from Onsager's critical exponents of the Ising model on a flat 2D regular lattice [10]. Due to quantum gravitational effects, the phase transition is changed from a second-order transition to a third-order transition at a finite critical temperature, and the continuum theory defined at the critical temperature turns out to be Liouville gravity coupled to a conformal field theory with central charge $c=1 / 2$. The scaling dimensions of primary operators in the $c=1 / 2$ conformal field theory change to the Knizhnik-Polyakov-Zamolodchikov values [11], which are caused by gravitational dressing. At the same time, the long range fluctuations of spins interacting with geometry changes the critical properties of 2D quantum gravity itself at the critical temperature.

\footnotetext{
*ambjorn@nbi.dk

†ysato@th.phys.nagoya-u.ac.jp

*omo@gravity.phys.waseda.ac.jp

${ }^{1}$ DT was first introduced as a regularization of 2D quantum gravity [3-8]. For a review, see [9].

Published by the American Physical Society under the terms of the Creative Commons Attribution 4.0 International license. Further distribution of this work must maintain attribution to the author(s) and the published article's title, journal citation, and DOI. Funded by SCOAP ${ }^{3}$.
}

Causal dynamical triangulations (CDT) were introduced as a different class of triangulations [12-14], mainly in an attempt to cure some of the problems encountered in the DT formalism in more than two dimensions (see $[15,16]$ for reviews). However, one can also study 2D CDT, and in particular one can couple the Ising spins to the model, in the same spirit as described above. This spin model has not been solved analytically, but it can be studied by hightemperature expansions and Monte Carlo simulations and the results are clear: the critical exponents of the Ising model coupled to CDT are identical to the Onsager exponents $[17,18]$. The 2D CDT model allows much less geometrical fluctuations than the 2D DT model, and the allowed fluctuations are not strong enough to change the Onsager exponents of the spin system. ${ }^{2}$ In particular, it has been shown that the ability to create baby universes are important for the change of the Onsager exponents, and this is explicitly forbidden in 2D CDT [20].

A generalized 2D CDT model (GCDT), which allowed the creation of a finite number of baby universes, was introduced first as a continuum theory [21,22] and later defined at the discrete level $[23,24]$. As clarified in [24], the difference between the lattice structures of GCDT and DT is the presence of a weight $\theta$ in GCDT that controls the number of baby universes, i.e., the number of local maxima of the distance labeling from a vertex picked up by hand for

\footnotetext{
${ }^{2}$ While the critical spin exponents remain the ones of the flat space, the backreaction of spins on the geometry in the case of more than two Ising models coupled the triangulations is quite strong. Thus, we have a kind of $c=1$ barrier even for CDT. This is confirmed by studying massless scalar fields coupled to CDT [19].
} 
the sake of convenience. In GCDT, at the discrete level, one can take the continuum limit of DT for a fixed $\theta>0$ where the number of baby universes diverges, while tuning $\theta \rightarrow 0$ one can reach the continuum limit of GCDT characterized by a finite number of baby universes, which includes the continuum CDT if there exits a unique local maximum, i.e., a global maximum, of the distance labeling.

While the graphs used to define GCDT at a discretized level can be considered as a relative small extension of the graphs used to define CDT, there exists a bijection between the ensemble of graphs defining GCDT and a set of graphs characterized by having a finite number of faces. The bijection is such that the number of baby universes in GCDT is precisely the number of faces in this other set of graphs [24]. These latter graphs thus consist of (infinitely) many tree subgraphs and a finite number of faces in a specific continuum limit corresponding to the continuum limit of GCDT. Note here that when it comes to aspects of pure 2D quantum gravity (i.e., gravity without matter fields), even though the lattice structures of these latter graphs and those of GCDT are quite different, since one just basically counts the entropy of the graphs, these two classes of graphs lead to the same theory due to the bijection. However, these two classes of graphs will not necessarily lead to the same theories when matter is coupled to the graphs. This is illustrated in the case of CDT. If one couples Ising spin to the (rather regular) graphs originally used to define CDT, the Ising model will behave more or less like Ising spins on a regular lattice and in particular there is a phase transition with Onsager critical exponents, as mentioned above. However, via bijection, these CDT graphs are mapped to graphs with just one face, i.e., they are basically tree graphs. It is known that Ising spins on tree graphs cannot be critical.

One advantage of studying GCDT through the graphs consisting of tree subgraphs and a finite number of faces is that there exists a one-matrix model with a cubic interaction and a tadpole term which allows us to introduce the parameter $\theta$ mentioned above and which can interpolate between DT and GCDT (realized on the set of graphs with a finite number of faces) [23,25]. It also allowed for an intuitive understanding of this interpolation in terms of an inhomogeneous lattice structure, as well as the possibility of new scaling limits using this inhomogeneous lattice structure [26].

It is possible to couple Ising spins to GCDT (realized via the set of graphs with a finite number of faces) in the spirit of Kazakov and Boulatov, using a two-matrix model. It was first done in [27]. ${ }^{3}$ The corresponding two-matrix model was explicitly solved and shown to be related to the Boulatov-Kazakov model in [28]. In the one-matrix

\footnotetext{
${ }^{3}$ This model is inequivalent to the Ising model on the original GCDT including a finite number of baby universes, since the information on Ising-spin configurations is not preserved through the bijection.
}

model [26], it was shown, as mentioned above, how a scaling parameter $\theta$ allowed one to reach the GCDT regime from the DT regime in the limit $\theta \rightarrow 0$. These considerations were extended to the Ising model in the two-matrix model of [28]. For a fixed scaling parameter $\theta>0$, the model can be mapped onto the Boulatov-Kazakov Ising model. The critical temperature of the Ising spins of the model is a function of $\theta$ and for $\theta \rightarrow 0$ this critical temperature also goes to zero (but for any $\theta>0$, it is mapped to the critical temperature of the Boulatov-Kazakov Ising model, which is of course independent of $\theta$ ). Thus, the limit $\theta \rightarrow 0$ is interesting, since the geometry of the triangulations might change from DT to GCDT. According to [26], there are even several ways to take the $\theta \rightarrow 0$ limits, leading to different ensembles of triangulations with different fractal properties, and consequently these different limits might also lead to different critical behavior of the Ising spins. This is the topic we want to study in this paper.

This paper is organized as follows. In Sec. II, we review the Boulatov-Kazakov Ising model in a self-contained manner. Section III is devoted to an introduction of the model [28] and in particular to explain useful tools we use in due course. We then study the critical behaviors of the system in Sec. IV, focusing especially on the zero temperature. Section V contains summary and discussion.

\section{BOULATOV-KAZAKOV ISING MODEL}

The Ising model on 2D DT was first introduced in the seminal paper by Kazakov [1], and with an external magnetic field added to the system, all the critical exponents of the model could be calculated analytically [2]. In this section, we give a short review of this model (the Boulatov-Kazakov Ising model) without an external magnetic field, using triangulations with the Ising spins placed in the center of the triangles, or equivalently $\phi^{3}$ graphs with the Ising spins placed at the vertices.

Let us first consider a closed, connected, planar graph $G$ consisting of vertices of degree 3 , and define the Ising model on the graph $G$,

$$
Z_{G}(\beta)=\sum_{\{\sigma\}} \prod_{\langle i, j\rangle} e^{\beta \sigma_{i} \sigma_{j}},
$$

where $\beta$ is the inverse temperature, $\sigma_{i}$ being \pm 1 a spin located at a vertex $i, \sum_{\{\sigma\}}$ a sum over all spin configurations, and $\prod_{\langle i, j\rangle}$ a product with respect to all nearestneighbor pairs of vertices. The Boulatov-Kazakov Ising model is given by a sum of (2.1) over all possible closed, connected, planar graphs,

$$
F_{\mathrm{BK}}(g, c)=\sum_{G} \frac{1}{|\operatorname{Aut}(G)|}\left(\left(\frac{\sqrt{c}}{1-c^{2}}\right)^{3 / 2} g\right)^{n(G)} Z_{G}(\beta),
$$


where $|\operatorname{Aut}(G)|$ is the order of automorphism group of $G$, $n(G)$ the total number of vertices in $G, g$ essentially a weight for each vertex, and an additional weight $\left(\sqrt{c} /\left(1-c^{2}\right)\right)^{3 / 2}$ enters to make a connection with a matrix model introduced in due course. Here $c$ and $g$ are related to the inverse temperature and the dimensionless cosmological constant $\lambda$ such that $c=e^{-2 \beta}$ and $g=e^{-\lambda}$, respectively. The $\phi^{3}$ graphs (or the dual triangulations) are all assumed to have spherical topology, and the sum over these graphs is the lattice version of the integration over 2D (spherical) geometries, in this way coupling quantum gravity to the Ising model.

The sum (2.2) can be rewritten as a sum over the number of vertices,

$$
F_{\mathrm{BK}}(g, c)=\sum_{n}\left(\left(\frac{\sqrt{c}}{1-c^{2}}\right)^{3 / 2} g\right)^{n} Z_{n}(\beta) .
$$

We can view $n$ as proportional to the volume (the area A) of $2 \mathrm{D}$ spacetime, since in the dual graph $n$ is the number of triangles, which we all consider having the same area proportional to $\varepsilon^{2}$, where $\varepsilon$ is the length of a link in the triangulations. Thus, $A(n) \propto n \cdot \varepsilon^{2}$, and $Z_{n}(\beta)$ can be understood as the partition function of the Ising model of a fixed (spacetime) volume, but dressed by quantum gravity since it contains effects coming from the sum over all possible spherical triangulations with a given $n$. The power series (2.3) is convergent since for $n \gg 1$,

$$
Z_{n}(\beta) \propto\left(1 / g_{k}(\beta)\right)^{n} n^{\gamma_{s}-3}(1+\mathcal{O}(1 / n)),
$$

where $\gamma_{s}$ is a universal constant known as the string susceptibility exponent. A finite radius of convergence $g_{k}(\beta)$, sometimes also called a critical coupling constant, essentially defines the free energy per vertex in the thermodynamic limit,

$f(\beta)=-\frac{1}{\beta} \lim _{n \rightarrow \infty} \frac{1}{n} \log Z_{n}(\beta)=\frac{1}{\beta} \log \left[\left(\frac{\sqrt{c}}{1-c^{2}}\right)^{3 / 2} g_{k}(\beta)\right]$,

and the critical coupling constant has been computed $[1,2]$,

$$
\begin{aligned}
g_{k}^{2}(\beta)= & \left(\rho^{2}-c^{2}\right) \frac{(1+\rho)(-1+2 c+\rho)}{8 \rho} \\
& +2 c\left(\frac{(1+\rho)(-1+2 c+\rho)}{8 \rho}\right)^{2},
\end{aligned}
$$

where $c=e^{-2 \beta}$. In the low-temperature regime,

$$
\rho=-\sqrt{\frac{1-2 c}{3}}
$$

while in the high-temperature regime,

$$
\begin{aligned}
\rho= & -\frac{(2 c(1-c) \sqrt{1-2 c}+c(2+c(c-4)))^{1 / 3}}{2} \\
& -\frac{c}{2}\left(1+\frac{c}{(2 c(1-c) \sqrt{1-2 c}+c(2+c(c-4)))^{1 / 3}}\right) .
\end{aligned}
$$

At the critical temperature $\beta_{k}^{-1}$ or equivalently

$$
c_{k}:=e^{-2 \beta_{k}}=\frac{2 \sqrt{7}-1}{27},
$$

the free energy and its first derivative are differentiable functions, but the specific heat, a second derivative of the free energy,

$$
C=-\beta^{2} \frac{\partial^{2}}{\partial \beta^{2}}(\beta f(\beta)),
$$

has a cusp (see Fig. 1). This is a signal of a third-order phase transition. As mentioned, introducing a magnetic field into the system, all critical exponents have been calculated: $\alpha=-1, \beta=1 / 2, \gamma=2, \delta=5, d \nu=3$ [2], and they are different from the flat-space Onsager exponents.

\section{A. Continuum limit}

The power series (2.3) become singular at $g=g_{k}$, and its singular behavior is characterized by the appearance of a fractional power in the expansion around $g_{k}$,

$$
\begin{aligned}
Z(g, c)= & c_{0}+c_{1}\left(g_{k}-g\right)+c_{2}\left(g_{k}-g\right)^{2} \\
& +c_{2-\gamma_{s}}\left(g_{k}-g\right)^{2-\gamma_{s}}+\cdots,
\end{aligned}
$$

where $c_{0}, c_{1}, c_{2}$, and $c_{2-\gamma_{s}}$ are numerical constants and $\gamma_{s}$, string susceptibility exponent, quantifies the singularity. We have $\gamma_{s}=-1 / 2$ at $\beta \neq \beta_{k}$ while it changes at $\beta_{k}$ to $-1 / 3$. As mentioned, this change is a result of the backreaction of spins on the geometries.

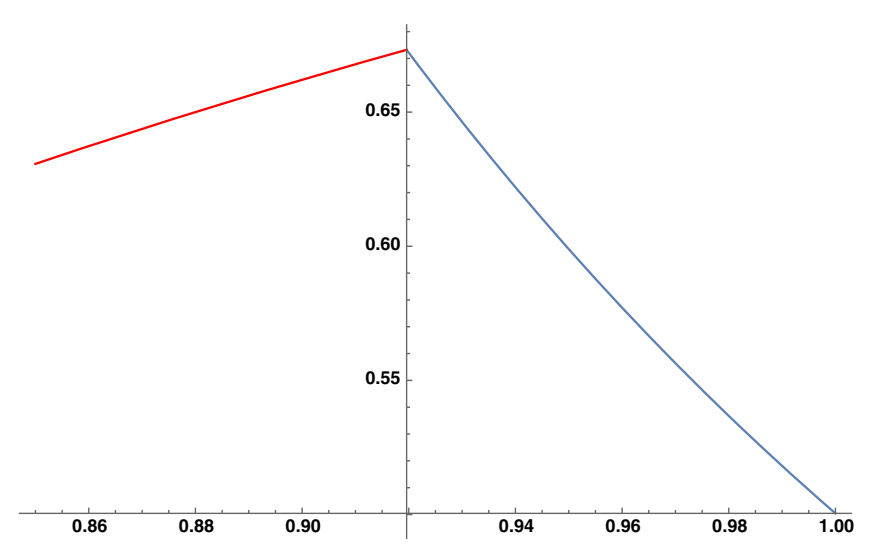

FIG. 1. A plot of the specific heat as a function of $\beta$. The curve has a cusp at $\beta_{k}$. 
The fractional power in the expansion implies that if one differentiates $Z(g, c)$ suitable times with respect to $g$, it diverges at $g=g_{k}$. As a result, the average number of vertices blows up when tuning $g$ to $g_{k}$,

$$
\langle n\rangle=\left.g \frac{\partial}{\partial g} \log Z(g, c)\right|_{\text {sing }} \sim \frac{1}{g_{k}-g},
$$

where "sing" means to pick up the singular part.

Using this singular behavior, one can define the continuum limit. The large- $n$ asymptotic behavior of the partition function (2.4) implies, for $n \gg 1$,

$$
g^{n} Z_{n}(\beta) \propto\left(g / g_{k}\right)^{n} n^{\gamma_{s}-3}=e^{-\left(\lambda-\lambda_{k}\right) n} n^{\gamma_{s}-3},
$$

where $g_{k}=: e^{-\lambda_{k}}$. As can be understood from (2.12), this large- $n$ behavior becomes important if tuning $g$ to $g_{k}$. Therefore, introducing the lattice spacing $\varepsilon$ of triangulations one can, as mentioned above, define the physical area $A$ and the renormalized cosmological constant $\Lambda$ by

$$
A=\varepsilon^{2} n, \quad \Lambda=\frac{\lambda-\lambda_{k}}{\varepsilon^{2}},
$$

and the continuum limit is obtained by tuning $g \rightarrow g_{k}$ (and correspondingly $\varepsilon \rightarrow 0$ ) such that $\Lambda$ is kept fixed and $A$ finite. Taking this continuum limit, one obtains

$$
g^{n} Z_{n}(\beta) \propto e^{-\Lambda A} A^{\gamma_{s}-3} .
$$

This quantity can be compared with the path integral of the Liouville theory coupled to conformal field theories with fixed area $A$. At $\beta \neq \beta_{k}$, it coincides with that of the Liouville theory for pure gravity, i.e., Liouville theory coupled to matter fields with $c=0$. At the critical temperature, the spin fluctuations diverge, and as a result one obtains instead the behavior of the Liouville theory coupled to a $c=1 / 2$ conformal field theory.

\section{B. The matrix model representation}

The so-called matrix models allow us to implement the sum over graphs via simple Gaussian integrals. In the case of the Boulatov-Kazakov Ising model, the following twomatrix model plays that role $[1,2]$ :

$$
Z_{N}(g, c)=\int D \psi_{+} D \psi_{-} e^{-N \operatorname{tr} V\left(\psi_{+}, \psi_{-}\right)},
$$

where $\psi_{ \pm}$are Hermitian $N \times N$ matrices, $D \psi_{ \pm}$the Haar measures on $\mathrm{U}(N)$, and the potential

$$
V\left(\psi_{+}, \psi_{-}\right)=\frac{1}{2} \psi_{+}^{2}+\frac{1}{2} \psi_{-}^{2}-c \psi_{+} \psi_{-}-\frac{g}{3}\left(\psi_{+}^{3}+\psi_{-}^{3}\right) .
$$

The integral (2.16) is defined formally as a power series with respect to $g$, and the coefficient to $g^{n}$ generates Feynman graphs with $n$ vertices of degree 3 . The vertices associated with $\psi_{+}^{3}$ can be thought of as having Ising spin $\sigma=1$ and the vertices associated with $\psi_{-}^{3}$ as having Ising spin $\sigma=-1$. By Wick's theorem, the integral (2.16) implements the sum over all possible graphs with the nearest-neighbor spin interactions taken into account properly if $c=e^{-2 \beta}$. If one takes the matrix size $N$ to be large, one can suppress nonplanar graphs in the sum. With this understanding, the Boulatov-Kazakov model can be defined by the matrix model as follows:

$$
F_{\mathrm{BK}}(g, c):=\lim _{N \rightarrow \infty} \frac{1}{N^{2}} \log \left(\frac{Z_{N}(g, c)}{Z_{N}(0, c)}\right),
$$

where the logarithm is needed to single out connected graphs.

\section{A SETUP FOR COOLING}

To reduce the critical temperature of the Ising model on 2D DT down to the zero temperature and examine its critical behavior, the following matrix model has been proposed [27,28]:

$$
I_{N}(g, c, \theta)=\int D \varphi_{+} D \varphi_{-} e^{-N \operatorname{tr} U\left(\varphi_{+}, \varphi_{-}\right)},
$$

where $\varphi_{ \pm}$are Hermitian $N \times N$ matrices, $D \varphi_{ \pm}$are the Haar measures on $\mathrm{U}(N)$, and the potential is given by

$$
\begin{aligned}
U\left(\varphi_{+}, \varphi_{-}\right)= & \frac{1}{\theta}\left(\frac{1}{2} \varphi_{+}^{2}+\frac{1}{2} \varphi_{-}^{2}-c \varphi_{+} \varphi_{-}-g\left(\varphi_{+}+\varphi_{-}\right)\right. \\
& \left.-\frac{g}{3}\left(\varphi_{+}^{3}+\varphi_{-}^{3}\right)\right) .
\end{aligned}
$$

Perturbative expansions with respect to $g$ give Feynman graphs consisting of vertices of degree 1 and 3. A typical planar graph is a skeleton graph with tree graphs attached (see the lhs of Fig. 2). Here the skeleton graph means a planar graph consisting only of vertices of degree 3 . The parameter $\theta$ is a loop-counting parameter meaning that if $\theta \ll 1$, loops in Feynman graphs are suppressed and tree structures become dominant. This kind of modification was first introduced in the context of a one-matrix model in order to obtain GCDT from DT and it defines a new continuum limit of one-matrix models $[23,25]$. The detailed disentanglement of the model in tree and skeleton graphs and possible scaling limits associated with this was studied in [26].

The two-matrix model (3.1) defines a slightly "modified" Ising model on 2D DT, 

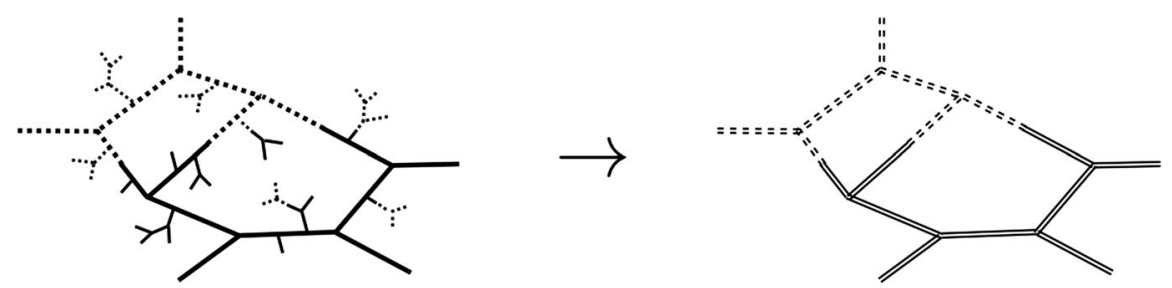

FIG. 2. The left figure: a typical planar graph generated by the potential (3.2) in which tree graphs attached to a skeleton graph. Each of the solid and dotted edges corresponds to the propagators $\left\langle\varphi_{+} \varphi_{+}\right\rangle_{0}$ and $\left\langle\varphi_{-} \varphi_{-}\right\rangle_{0}$, respectively; each of the half-solid and half-dotted edges corresponds to the propagator, $\left\langle\varphi_{+} \varphi_{-}\right\rangle_{0}$ or $\left\langle\varphi_{-} \varphi_{+}\right\rangle_{0}$. The right figure: a typical planar graph generated by the potential (3.9) in which all tree graphs are integrated out. Each of the solid double and dotted double lines corresponds to the propagators $\left\langle\tilde{\varphi}_{+} \tilde{\varphi}_{+}\right\rangle_{0}$ and $\left\langle\tilde{\varphi}_{-} \tilde{\varphi}_{-}\right\rangle_{0}$ respectively; each half-solid and half-dotted double line corresponds to the propagator, $\left\langle\tilde{\varphi}_{+} \tilde{\varphi}_{-}\right\rangle_{0}$ or $\left\langle\tilde{\varphi}_{-} \tilde{\varphi}_{+}\right\rangle_{0}$.

$$
\begin{aligned}
F_{\mathrm{ST}}(g, c, \theta):= & \lim _{N \rightarrow \infty} \frac{1}{N^{2}} \log \left(\frac{I_{N}(g, c, \theta)}{I_{N}(0, c, \theta)}\right) \\
= & \sum_{G} \frac{1}{|\operatorname{Aut}(G)|}\left(\left(\frac{\sqrt{c}}{1-c^{2}}\right)^{1 / 2} \frac{g}{\sqrt{\theta}}\right)^{n_{1}(G)} \\
& \times\left(\left(\frac{\sqrt{c}}{1-c^{2}}\right)^{3 / 2} g \sqrt{\theta}\right)^{n_{3}(G)} Z_{G}(\beta),
\end{aligned}
$$

where $G$ denotes a closed, connected, planar graph generated by the matrix model with the potential (3.2), and $n_{1}$ and $n_{3}$ the total number of vertices of degree 1 and 3 , respectively. The critical temperature of this system becomes a function of $\theta$, and as shown in [28], when $\theta \neq 0$, the critical behavior is nothing but that of the Boulatov-Kazakov Ising model, but when tuning $\theta \rightarrow 0$ the critical temperature reaches zero temperature at which the tree structures become dominant, resulting in a continuum theory different from the Liouville theory coupled to a conformal matter with $c=1 / 2$.

It is useful to classify vertices in a graph $G$ into two kinds: skeleton vertices and others. Let us pick up a vertex of degree 3 and label the three links emanating from the vertex, say 1,2 , and 3 in a clockwise manner. When moving from that vertex to other vertices via links, if one can find a path coming back to the first vertex whichever link 1,2, or 3, one starts with, the vertex picked up is called a skeleton vertex. Since $\theta$ is a loop-counting parameter, the number of skeleton vertices is supposed to be controlled by $\theta$. Concerning (3.3), if we implement the redefinition

$$
n_{t}(G):=n_{1}(G)+n_{3}(G), \quad n_{s}(G):=n_{3}(G)-n_{1}(G),
$$

where $n_{t}(G)$ and $n_{s}(G)$ are the total number of vertices and the number of skeleton vertices in a given graph $G$, we obtain

$$
F_{\mathrm{ST}}(g, c, \theta)=\sum_{G} \frac{1}{|\operatorname{Aut}(G)|} g_{t}^{n_{t}(G)} g_{s}^{n_{s}(G)} Z_{G}(\beta)
$$

where

$$
g_{t}=\frac{\sqrt{c}}{1-c^{2}} g, \quad g_{s}=\left(\frac{\sqrt{c}}{1-c^{2}}\right)^{1 / 2} \sqrt{\theta}
$$

From (3.5) and (3.6), it is indeed $\theta$ that controls the number of skeleton vertices, while the total number of vertices is controlled by $g$.

\section{A. Relation to the Boulatov-Kazakov Ising model}

As shown in [28], ${ }^{4}$ one can map our matrix model defined by (3.3) to the matrix model for the BoulatovKazakov Ising model (2.16). This relation turns out to be useful for understanding the physics of our Ising model.

Starting from the matrix model (3.3) and changing variables

$$
\begin{aligned}
\varphi_{ \pm} & =\tilde{\varphi}_{ \pm}+Z_{\text {tree }}(g, c), \quad \text { with } \\
Z_{\text {tree }}(g, c) & =\frac{1-c-\sqrt{(1-c)^{2}-4 g^{2}}}{2 g},
\end{aligned}
$$

the integral (3.3) becomes

$I_{N}(g, c, \theta)=e^{N^{2} F_{\text {cons }}(g, c, \theta)} \int D \tilde{\varphi}_{+} D \tilde{\varphi}_{-} e^{-N \operatorname{tr} \tilde{U}\left(\tilde{\varphi}_{+}, \tilde{\varphi}_{-}\right)}$,

where

$$
\begin{aligned}
\tilde{U}\left(\tilde{\varphi}_{+}, \tilde{\varphi}_{-}\right)= & \frac{1}{\theta}\left(\frac{1-2 g Z_{\text {tree }}(g, c)}{2}\left(\tilde{\varphi}_{+}^{2}+\tilde{\varphi}_{-}^{2}\right)\right. \\
& \left.-c \tilde{\varphi}_{+} \tilde{\varphi}_{-}-\frac{g}{3}\left(\tilde{\varphi}_{+}^{3}+\tilde{\varphi}_{-}^{3}\right)\right)
\end{aligned}
$$

\footnotetext{
${ }^{4}$ In the one-matrix model case, a similar transformation has been considered in [26].
} 


$$
\begin{aligned}
F_{\text {cons }}(g, c, \theta)= & \frac{1}{6 \theta g^{2}}\left(1-c^{3}-\sqrt{(1-c)^{2}-4 g^{2}}\right. \\
& -g^{2}\left(6-4 \sqrt{(1-c)^{2}-4 g^{2}}\right) \\
& -c\left(3-6 g^{2}-2 \sqrt{(1-c)^{2}-4 g^{2}}\right) \\
& \left.+c^{2}\left(3-\sqrt{(1-c)^{2}-4 g^{2}}\right)\right) .
\end{aligned}
$$

Here $Z_{\text {tree }}$ is the sum of all connected planar, rooted tree graphs with a spin placed at each vertex of degree 1 and 3, as well as the sum over all spin configurations [28].

Through this transformation, the linear terms in (3.2) are integrated out and a typical Feynman graph is depicted in the rhs of Fig. 2, which is a skeleton graph with dressed edges. The noncanonical quadratic terms in (3.9) contribute to the dressed edges through the dressed propagators

$$
\begin{aligned}
\left\langle\tilde{\varphi}_{ \pm} \tilde{\varphi}_{ \pm}\right\rangle_{0} & =\frac{1-2 g Z_{\text {tree }}}{\left(1-2 g Z_{\text {tree }}\right)^{2}-c^{2}}\left\langle\varphi_{ \pm} \varphi_{ \pm}\right\rangle_{0}, \quad \text { with } \\
\left\langle\varphi_{ \pm} \varphi_{ \pm}\right\rangle_{0} & =\frac{\theta}{N}
\end{aligned}
$$

$\left\langle\tilde{\varphi}_{ \pm} \tilde{\varphi}_{\mp}\right\rangle_{0}=\frac{1}{\left(1-2 g Z_{\text {tree }}\right)^{2}-c^{2}}\left\langle\varphi_{ \pm} \varphi_{\mp}\right\rangle_{0}, \quad$ with

$\left\langle\varphi_{ \pm} \varphi_{\mp}\right\rangle_{0}=\frac{\theta c}{N}$,

where the indices of the matrices have been omitted. The dressed propagators (3.11) and (3.12) can be obtained by summing all possible tree outgrowths from the canonical propagators, $\left\langle\varphi_{ \pm} \varphi_{ \pm}\right\rangle_{0}$ and $\left\langle\varphi_{ \pm} \varphi_{\mp}\right\rangle_{0}$ [28].

Rescaling the new variables,

$$
\tilde{\varphi}_{ \pm}=\sqrt{\frac{\theta}{1-2 g Z_{\text {tree }}(g, c)}} \psi_{ \pm},
$$

the integral (3.8) becomes

$$
\begin{aligned}
I_{N}(g, c, \theta)= & \left(\frac{\theta}{c+\sqrt{(1-c)^{2}-4 g^{2}}}\right)^{N^{2}} \\
& \times e^{N^{2} F_{\text {cons }}(g, c, \theta)} Z_{N}\left(c_{\mathrm{BK}}, g_{\mathrm{BK}}\right),
\end{aligned}
$$

where $Z_{N}$ is nothing but the matrix model for the BoulatovKazakov Ising model, with the coupling constants $(g, c)$ substituted by the "Boulatov-Kazakov" coupling constants $\left(c_{\mathrm{BK}}, g_{\mathrm{BK}}\right)$ defined by

$$
c_{\mathrm{BK}}=\frac{c}{1-2 g Z_{\text {tree }}(g, c)}=\frac{c}{c+\sqrt{(1-c)^{2}-4 g^{2}}},
$$

$$
g_{\mathrm{BK}}=\frac{\theta^{1 / 2} g}{\left(1-2 g Z_{\text {tree }}(g, c)\right)^{3 / 2}}=\frac{\theta^{1 / 2} g}{\left(c+\sqrt{(1-c)^{2}-4 g^{2}}\right)^{3 / 2}} .
$$

As a result, we obtain

$F_{\mathrm{ST}}(g, c, \theta)=F_{\mathrm{BK}}\left(g_{\mathrm{BK}}, c_{\mathrm{BK}}\right)+F_{\text {tree }}(g, c)+F_{\text {cons }}(g, c, \theta)$,

where

$$
F_{\text {tree }}(g, c)=-\log \left[c+\sqrt{(1-c)^{2}-4 g^{2}}\right] .
$$

Through the change of matrix variables, the inverse temperature in our original system, $\beta=-\log [c] / 2$, changes to

$$
\begin{aligned}
\beta_{\mathrm{BK}} & =-\frac{1}{2} \log \left[c_{\mathrm{BK}}(g, c)\right] \\
& =\beta\left(1-\frac{\log \left[c+\sqrt{(1-c)^{2}-4 g^{2}}\right]}{\log [c]}\right) .
\end{aligned}
$$

We can think of this change of temperature as a change of spins, i.e., the nearest-neighbor spin interaction changes if integrating trees out,

$$
-\beta \sum_{\langle i, j\rangle \in v(G)} \sigma_{i} \sigma_{j} \Rightarrow-\beta_{\mathrm{BK}} \sum_{\langle i, j\rangle \in v\left(G_{s}\right)} \sigma_{i} \sigma_{j},
$$

where $\langle i, j\rangle \in v(G) \quad\left(\langle i, j\rangle \in v\left(G_{s}\right)\right)$ denotes a pair of nearest-neighbor vertices $i$ and $j$ in a set of vertices in a graph $G\left(G_{s}\right.$, a skeleton graph), and then we can define effective spins $\tilde{\sigma}_{i}(g, c)$ 's by the following equation:

$-\beta_{\mathrm{BK}} \sum_{\langle i, j\rangle \in v\left(G_{s}\right)} \sigma_{i} \sigma_{j}=:-\beta \sum_{\langle i, j\rangle \in v\left(G_{s}\right)} \tilde{\sigma}_{i}(g, c) \tilde{\sigma}_{j}(g, c)$,

where

$\tilde{\sigma}_{i}(g, c)=\left(1-\frac{\log \left[c+\sqrt{(1-c)^{2}-4 g^{2}}\right]}{\log [c]}\right)^{1 / 2} \sigma_{i}=: \sqrt{z} \sigma_{i}$.

One can show that $0 \leq \sqrt{z} \leq 1$ if $g \leq(1-c) / 2$ for a given $c$. A qualitative behavior of the "spin renormalization" $\sqrt{z}$ can be seen in Fig. 3 .

At zero temperature $c=0$, we have $\sqrt{z}=1$ for $g \leq 1 / 2$. On the other hand, at high temperature $c=1$, we have $g=0$ and $\sqrt{z}=0$. As one can see from Fig. 3, the effective spins behave like ordinary spins in the low-temperature regime since the spin renormalization is not that sensitive to $g$ and its value is almost 1 . However, in the 


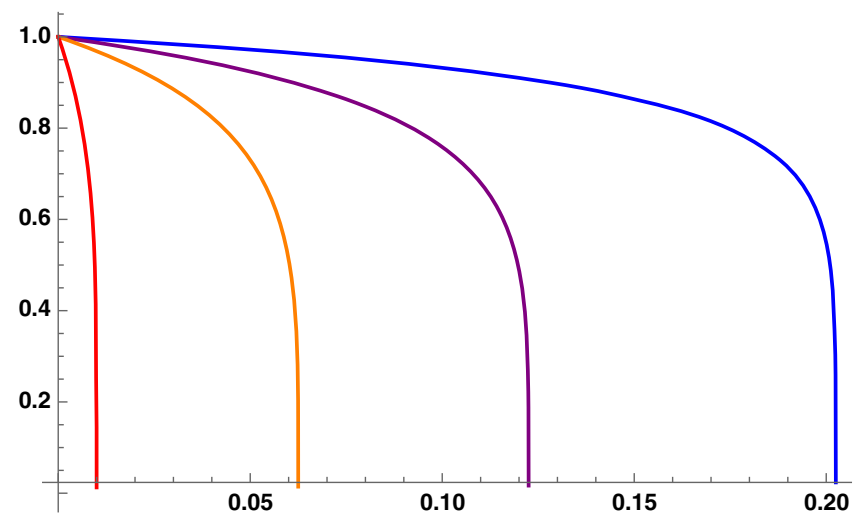

FIG. 3. Plots of $\sqrt{z}$ as a function of $g^{2}$ for a given value of $c$. Blue, purple, orange, and red curves are those for $c=0.1,0.3$, $0.5,0.8$, respectively.

high-temperature regime, the spin renormalization is very sensitive to $g$ and rapidly goes down when increasing $g$ up to its critical value $(1-c) / 2$. This means that at high temperature, the effective spins strongly "feel" the existence of tree structures (already integrated out) which weaken the effective spins.

\section{CRITICALITY}

In this section, we study the critical behavior of our Ising model defined in terms of the matrix model (3.1), focusing especially on the zero-temperature regime.

The critical curve on which spin fluctuations diverge is determined by the set of equations [28],

$$
\begin{gathered}
1-4 g_{k}^{2}=\frac{2^{2 / 3}}{5^{1 / 3}}\left(\theta g_{k}^{2}\right)^{1 / 3}\left(1+\frac{27}{2 \times 10^{1 / 3}}\left(\theta g_{k}^{2}\right)^{1 / 3}\right), \\
c_{k}=\frac{1}{10^{1 / 3}}\left(\theta g_{k}^{2}\right)^{1 / 3} .
\end{gathered}
$$

Removing $\theta$ from (4.1) and (4.2), we find the critical curve

$$
c_{k}=\frac{2 \sqrt{7-27 g_{k}^{2}}-1}{27} .
$$

Inserting (4.3) into (3.15), we recover the critical point obtained by [2]

$$
\left(c_{\mathrm{BK}}\right)_{k}=\frac{2 \sqrt{7}-1}{27}, \quad\left(g_{\mathrm{BK}}\right)_{k}=\frac{\sqrt{10}}{(1+2 \sqrt{7})^{3 / 2}} .
$$

Therefore, on the critical curve (4.3) except at the end point $\theta=0$, we should observe the same criticality as that of the Boulatov-Kakakov Ising model (4.4). In this sense, let us call the curve (4.3) the Boulatov-Kazakov critical curve. The reason the critical point of the Boulatov-Kazakov Ising model has been replaced by a curve is that our model has the additional parameter $\theta$, and we can use this parameter in a parametric representation of the Boulatov-Kazakov critical curve. From (4.1) and (4.2), we can determine $c_{k}$ and $g_{k}$ as functions of $\theta$ [28],

$$
\begin{aligned}
& c_{k}(\theta)=\frac{\theta^{1 / 3}}{10^{1 / 3}}\left(-\frac{9 \theta^{2 / 3}}{4 \times 10^{2 / 3}}+\frac{3^{1 / 3} \theta^{1 / 3}(243 \theta-80)+H^{2}}{4 \times 30^{2 / 3} H}\right), \\
& g_{k}(\theta)=\left(-\frac{9 \theta^{2 / 3}}{4 \times 10^{2 / 3}}+\frac{3^{1 / 3} \theta^{1 / 3}(243 \theta-80)+H^{2}}{4 \times 30^{2 / 3} H}\right)^{3 / 2},
\end{aligned}
$$

where

$$
\begin{aligned}
H= & {[81(40-81 \theta) \theta} \\
& +80(90+\sqrt{8100+3(2510-5103 \theta) \theta})]^{1 / 3} .
\end{aligned}
$$

In addition, we have another critical curve for dominant trees determined by the condition that the average number of vertices in the dressed propagators (3.11) and (3.12),

$$
g \frac{\partial}{\partial g} \log \left\langle\tilde{\varphi}_{ \pm} \tilde{\varphi}_{ \pm}\right\rangle_{0}, \quad g \frac{\partial}{\partial g} \log \left\langle\tilde{\varphi}_{ \pm} \tilde{\varphi}_{\mp}\right\rangle_{0}
$$

diverge, which yields

$$
c=1-2 g .
$$

Inserting (4.9) into (3.15) and (3.16), we have

$$
c_{\mathrm{BK}}=1, \quad g_{\mathrm{BK}}=\frac{g \sqrt{\theta}}{(1-2 g)^{3 / 2}} .
$$

From (2.6),

$$
\left(g_{\mathrm{BK}}\right)_{k}\left(c_{\mathrm{BK}}=1\right)=0 .
$$

Therefore, we conclude that $\theta=0$ on the critical curve for dominant trees.

We can compute the free energy per vertex on the critical curve for dominant trees. Let us rewrite (3.5) as

$$
F_{\mathrm{ST}}(g, c, \theta)=\sum_{n_{t}, n_{s}} g_{t}^{n_{t}} g_{s}^{n_{s}} Z_{n_{t}, n_{s}}(\beta)=\sum_{n_{s}} g_{s}^{n_{s}} \sum_{n_{t}} g_{t}^{n_{t}} Z_{n_{t}, n_{s}}(\beta) .
$$

For a given finite $n_{s}$, the radius of convergence for the power series of $g_{t}$ yields

$$
\left(g_{t}\right)_{k}(\beta)=\frac{g_{*} \sqrt{c}}{1-c^{2}}=\frac{\sqrt{c}}{2(1+c)},
$$




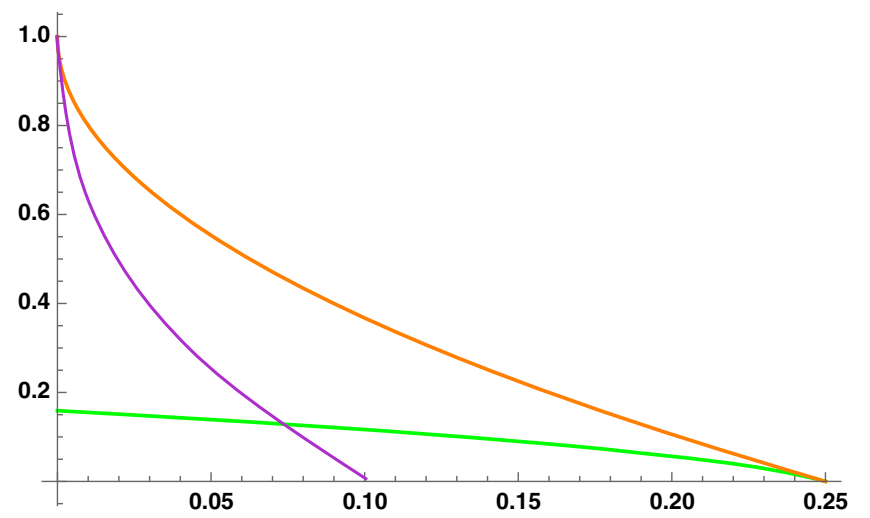

FIG. 4. The phase diagram: The vertical axis is $c$ and the horizontal one is $g^{2}$. The green curve is the Boulatov-Kazakov critical curve that separates the high- and low-temperature phases, and the orange curve is the critical curve for dominant trees. The purple curve is a schematic critical curve for the Liouville gravity for a fixed $\theta$.

where we have used (3.6) and $g_{*}$ is a solution to (4.9). The free energy per vertex on the critical curve for dominant trees is

$$
\begin{aligned}
f_{\text {tree }}(\beta) & =-\frac{1}{\beta} \lim _{n_{t} \rightarrow \infty} \frac{1}{n_{t}} \log Z_{n_{t}, n_{s}}(\beta)=\frac{1}{\beta} \log \left[\left(g_{t}\right)_{k}(\beta)\right] \\
& =-\frac{1}{\beta} \log (4 \cosh [\beta]) .
\end{aligned}
$$

This is essentially the free energy per vertex of the classical 1D spin chain, or that of the Ising model on branched polymers [29]. Thus, along the critical curve for dominant trees, the system is magnetized only at zero temperature.

Figure 4 is the phase diagram. For a fixed $\theta>0$, we have a well-defined map given by Eqs. (3.15) and (3.16) between $(g, c)$ and $\left(g_{\mathrm{BK}}, c_{\mathrm{BK}}\right)$. In the Boulatov-Kazakov model, we have a critical curve: for a given temperature $\beta_{\mathrm{BK}}^{-1}$, i.e., a given $c_{\mathrm{BK}}=e^{-2 \beta_{\mathrm{BK}}}$, there is a corresponding critical $g_{\mathrm{BK}}$ where the continuum limit is that of Liouville gravity. This defines the critical curve in the $\left(g_{\mathrm{BK}}, c_{\mathrm{BK}}\right)$ plane. One point on this curve is special, namely, the point given by Eq. (4.4), where the Liouville gravity theory changes from pure 2D gravity to $2 \mathrm{D}$ gravity coupled to a $c=1 / 2$ conformal theory. For a fixed value of $\theta>0$, one can draw the corresponding curve in the $(g, c)$ plane. This is the purple curve in Fig. 4. For a fixed $\theta$, this is where one can take the continuum limit in our modified model. It crosses the Boulatov-Kazakov critical curve where the Ising spins become critical. For each $\theta$, one has a different curve. The vertical axis in the phase diagram corresponds to the case with $\theta$ being $\infty$, and with decreasing $\theta$ the curves gradually move to the right in such a way that they share the point $\left(g^{2}, c\right)=(0,1)$ and asymptotically approach the critical curve for dominant trees on which $\theta=0$. To reach the zero-temperature critical point at which two kinds of critical lines meet, one has to tune $\theta$ to 0 .

\section{A. Zero-temperature criticality}

The physics around the zero-temperature critical point is very sensitive to the way we approach the point. In the following, we show several examples.

One way to approach the zero-temperature critical point, proposed in [28], is

$$
g(\theta)=g_{k}(\theta)\left(1-\varepsilon^{2} \Lambda\right), \quad c(\theta)=c_{k}(\theta), \quad \theta=\varepsilon^{3} \Theta,
$$

where $\Lambda$ and $\Theta$ are dimension-full coupling constants. $g(\theta)$ is chosen to be slightly away from the Boulatov-Kazakov critical curve in the spirit of (2.14), allowing us to interpret $\Lambda$ as a cosmological constant, while the temperature is chosen such that tuning $g(\theta) \rightarrow g_{k}(\theta)$ for fixed $\theta$ we would obtain Liouville gravity coupled to conformal matter with $c=1 / 2$. However, here we now take the limit where $\varepsilon \rightarrow 0$, i.e., $\theta$ is scaled to 0 at the same time as $g(\theta) \rightarrow g_{k}(\theta)$. This limit defines a continuum theory around the zero-temperature critical point that is described by a continuum two-matrix model [28]. To analyze this limit (4.14), let us introduce

$$
\begin{gathered}
\frac{c_{\mathrm{BK}}}{\left(c_{\mathrm{BK}}\right)_{k}}=\frac{c}{c_{k}}\left(\frac{c_{k}+\sqrt{\left(1-c_{k}\right)^{2}-4 g_{k}^{2}}}{c+\sqrt{(1-c)^{2}-4 g^{2}}}\right), \\
\frac{g_{\mathrm{BK}}}{\left(g_{\mathrm{BK}}\right)_{k}}=\frac{g}{g_{k}}\left(\frac{c_{k}+\sqrt{\left(1-c_{k}\right)^{2}-4 g_{k}^{2}}}{c+\sqrt{(1-c)^{2}-4 g^{2}}}\right)^{3 / 2},
\end{gathered}
$$

which have been obtained from (3.15) and (3.16). Plugging (4.14) into (4.15) and (4.16), one obtains at the small- $\varepsilon$ limit

$$
\begin{gathered}
\frac{c_{\mathrm{BK}}}{\left(c_{\mathrm{BK}}\right)_{k}}=\frac{1+2 \sqrt{7}}{1+2 \sqrt{7+5^{2 / 3} \frac{\Lambda}{\Theta^{2 / 3}}}}, \\
\frac{g_{\mathrm{BK}}}{\left(g_{\mathrm{BK}}\right)_{k}}=\left(\frac{1+2 \sqrt{7}}{1+2 \sqrt{7+5^{2 / 3} \frac{\Lambda}{\Theta^{2 / 3}}}}\right)^{3 / 2} .
\end{gathered}
$$

Therefore, with the continuum limit (4.14), spins on skeleton graphs cannot be critical and the criticality is governed by that of trees.

Next, we introduce a continuum limit such that spins, tree graphs, and skeleton graphs are all critical, which can be realized by the following choice: 


$$
\begin{aligned}
g(\theta) & =g_{k}(\theta)\left(1-\varepsilon^{2} \Lambda\right), \quad c(\theta)=c_{k}(\theta), \\
\theta & =\varepsilon^{a} \Theta_{a}, \quad \text { with } \quad 0<a<3,
\end{aligned}
$$

where $\Theta_{a}$ is a dimension-full coupling constant. This $a$-dependent continuum limit has been first introduced in the context of one-matrix model [26]. Inserting (4.19) into (4.15) and (4.16), one obtains

$$
\begin{gathered}
\frac{c_{\mathrm{BK}}}{\left(c_{\mathrm{BK}}\right)_{k}}=1-\frac{5^{2 / 3}}{14+\sqrt{7}} \frac{\Lambda}{\Theta_{a}^{2 / 3}} \varepsilon^{2-\frac{2}{3} a}+\cdots, \\
\frac{g_{\mathrm{BK}}}{\left(g_{\mathrm{BK}}\right)_{k}}=1-\frac{3 \times 5^{2 / 3}}{2(14+\sqrt{7})} \frac{\Lambda}{\Theta_{a}^{2 / 3}} \varepsilon^{2-\frac{2}{3} a}+\cdots .
\end{gathered}
$$

This means that if $0<a<3$, spins, tree graphs and skeleton graphs are all critical. Based on the parametrization (4.19), we can show

$$
n_{t} \sim \frac{1}{\varepsilon^{2}}, \quad n_{p} \sim \frac{1}{\varepsilon^{2 a / 3}}, \quad n_{s} \sim \frac{1}{\varepsilon^{2-2 a / 3}},
$$

where $n_{t}, n_{p}$, and $n_{s}$ are the total number of vertices, the number of vertices in the dressed propagators, and the number of skeleton vertices.

\section{B. New critical exponents}

Let us first consider the approach to zero temperature given by Eq. (4.14). Recall that the whole critical line of Fig. 4 is mapped to the single critical point $\left(\left(g_{\mathrm{BK}}\right)_{k},\left(c_{\mathrm{BK}}\right)_{k}\right)$ for the Boulatov-Kazakov Ising model on skeleton graphs. Similarly, the line defined by (4.14) is (for small $\varepsilon$ ) mapped to a single point $\left(g_{\mathrm{BK}}, c_{\mathrm{BK}}\right)$ given by Eqs. (4.17) and (4.18). This point has a finite distance (not necessarily small) to $\left(\left(g_{\mathrm{BK}}\right)_{k},\left(c_{\mathrm{BK}}\right)_{k}\right)$, and the Ising spins on the (finite number of) skeleton vertices are uncorrelated. On the other hand, for the tree graph related to a given link (the dressed propagators), the number of vertices is of the order $1 / \varepsilon^{2}$ while the temperature $\beta^{-1}$ is determined by $c=e^{-2 \beta}$ and $c \propto \theta^{1 / 3} \propto \varepsilon$. Since the tree graph has Hausdorff dimension two and thus linear extension of order $1 / \varepsilon$, and since spin correlations in the tree behave essentially like on a linear chain, the correlation length is $\xi(\beta)=1 / c$. Thus, we see that a given tree is essentially magnetized. ${ }^{5}$ A given spin configuration thus looks amazingly like a real, unmagnetized ferromagnetic material: it consists of a number of essentially magnetized regions (the dressed propagators), but the orientation of these is quite random, and the total magnetization of the piece of material is close to zero.

\footnotetext{
${ }^{5}$ By magnetized, we mean that the spins for a given configuration will essentially be aligned. However, since the volume is finite $\left(1 / \varepsilon^{2}\right.$ for the tree), the ensemble average will have zero magnetization, as is well known for the linear spin chain at nonzero temperature.
}

However, we should stress that while there is this resemblance with a ferromagnet for a single configuration, the statistical properties are quite different, since a tree never is magnetized for nonzero temperature. The magnetic properties are like those of a one-dimensional Ising chain as we have shown explicitly.

Let us now turn to the more nontrivial scaling given by Eq. (4.19) and characterized by the parameter $a$ between 0 and 3. For a given dressed propagator the situation is as before: the size of a typical tree associated with a dressed propagator is according to (4.22) $n_{p} \sim 1 / \varepsilon^{2 a / 3}$ and $c(\varepsilon) \sim \varepsilon^{a / 3}$, i.e., the correlation length $\xi(\varepsilon) \sim 1 / \varepsilon^{a / 3}$. Thus, a tree in a given configuration is essentially magnetized. However, according to (4.22), we have now a divergent number of skeleton vertices for $\varepsilon \rightarrow 0$ and the corresponding Boulatov-Kazakov coupling constants $\left(g_{\mathrm{BK}}(\varepsilon), c_{\mathrm{BK}}(\varepsilon)\right)$ now approach $\left(\left(g_{\mathrm{BK}}\right)_{k},\left(c_{\mathrm{BK}}\right)_{k}\right)$ for $\varepsilon \rightarrow 0$. The critical spin properties in such an approach is governed by two factors. The first one is that $c_{\mathrm{BK}}(\varepsilon) \neq\left(c_{\mathrm{BK}}\right)_{k}$. Thus, even if we had an infinite number of skeleton vertices, we would only have a finite spin correlation length

$$
\xi(\Delta c) \sim \frac{1}{|\Delta c|^{\nu}}, \quad \Delta c=\left(c_{\mathrm{BK}}\right)_{k}-c_{\mathrm{BK}},
$$

and approaching the critical point from the low-temperature phase as we are doing, we would have a magnetization per skeleton vertex,

$$
m(\Delta c) \sim|\Delta c|^{\beta}
$$

where the critical exponent $\beta$ should not be confused with the inverse temperature also called $\beta$. This magnetization per skeleton vertex would be present if we had an infinite volume, i.e., if we for the given $c_{\mathrm{BK}}(\varepsilon)$, instead of $g_{\mathrm{BK}}(\varepsilon)$, had chosen the critical $\left(g_{\mathrm{BK}}\right)_{k}$ corresponding to $c_{\mathrm{BK}}(\varepsilon)$. However, we have a $g_{\mathrm{BK}}(\varepsilon) \neq\left(g_{\mathrm{BK}}\right)_{k}$, and thus we have a finite number of skeleton vertices $n_{s} \sim 1 / \varepsilon^{2-2 \alpha / 3}$, i.e., a finite volume $V_{s}$. In a finite volume, we cannot determine the critical point $\left(c_{\mathrm{BK}}\right)_{k}$ but we can determine the $c_{\mathrm{BK}}$ where the correlation length $\xi$ becomes of the order of the size of the system, or expressed in terms of the volume $V_{s}$ : $\xi^{d}=V_{s}$, where $d$ is the dimension of the system. ${ }^{6}$ According to (4.23), this corresponds to a $\Delta c$ of the order

$$
\begin{aligned}
\Delta c & =\frac{1}{V_{s}^{1 / d \nu}}, \\
d \nu & =3 \text { for the Boulatov-Kazakov model. }
\end{aligned}
$$

\footnotetext{
${ }^{6}$ For a regular lattice, the meaning of $d$ is clear, but for DT lattices the meaning is less clear. However, we will not need a precise definition since only the combination $\nu d$ enters in the discussion, and if hyperscaling is valid, then $\nu d$ is related to the critical exponent for the specific heat $\alpha=2 / \nu d$.
} 
We now have two $\Delta c$, which are functions of $\varepsilon$,

$$
\begin{aligned}
& \Delta_{1} c=\left(c_{\mathrm{BK}}\right)_{k}-c_{\mathrm{BK}}(\varepsilon) \sim \varepsilon^{2-2 a / 3} \sim \frac{1}{V_{s}(\varepsilon)}, \quad \text { and } \\
& \Delta_{2} c=\left(\frac{1}{V_{s}(\varepsilon)}\right)^{1 / d \nu}=\left(\frac{1}{V_{s}(\varepsilon)}\right)^{1 / 3} .
\end{aligned}
$$

Clearly, $\Delta_{1} c$ is irrelevant for the way we have chosen to approach zero temperature. Inserting into (4.24), we obtain

$$
m(\varepsilon) \sim V_{s}^{-\beta / d \nu}=V_{s}^{-1 / 6},
$$

which is just the standard result for the Boulatov-Kazakov model. However, viewed from outside where we do not insist in resolving the graphs in dressed propagators and skeleton graphs, the dressed propagators do not in average contribute to the total spin, since this is already included in the mapping to the Boulatov-Kazakov model as emphasized in Eq. (3.20). If we denote the number of vertices in the complete graph by $V$, we can write magnetization per vertex in the complete graph,

$$
m^{\prime}(\varepsilon) \sim m(\varepsilon) \frac{V_{s}}{V} \sim \frac{1}{V^{(1+5 a / 3) / 6}} .
$$

This is a new kind of critical behavior which interpolates between the Boulatov-Kazakov model, $a=0$, and the GCDT model $a=3$. However, in order to be able to identify the scaling behavior with a critical exponent $\tilde{\beta}$, like in Eq. (4.24), we have to write it in the form $V^{-\tilde{\beta} / \tilde{\nu}}$, and it is unclear how to think about exponents $\tilde{v}$ and $\tilde{d}$ in our model. Hyperscaling usually links $\nu d$ to the exponent $\alpha$ for the specific heat by $\alpha=2-\nu d$. However, in our model, there is no natural definition of the dimension $d$ valid at all scales: the trees have a fractal dimension that is different from fractal dimension of the skeleton graph, so it seems unlikely that such a hyperscaling relation exists.

\section{DISCUSSION}

The DT graphs used to regularize 2D quantum gravity have generic fractal properties, among those that the fractal dimension of the graphs is four [30]. The generalized causal triangulations is another wide set of graphs, characterized by the property that the (graph)-distance from a vertex only has a finite number of local maxima, even for infinite graphs. The GCDT graphs have fractal dimension two. The coupling of matter to the ensembles of graphs is reasonably well understood in the DT case. If the matter system becomes critical for a certain choice of coupling constants, it defines a conformal field theory coupled to $2 \mathrm{D}$ gravity and both the critical properties of that matter system and the fractal properties of the ensemble of graphs change.

Coupling of matter theories to GCDT graphs are much less studied, but the interaction between graphs and matter does not seem to change the critical properties of graphs or of the matter systems (if they have critical couplings). This is in agreement with the general expectation that changes of the critical properties are caused by infinitely many baby universes. When coupling the Ising model to GCDT for instance, Onsager's critical exponents are expected to be recovered and the fractal dimension is expected to be two in the continuum limit characterized by a finite number of baby universes. However, so far one has not been able to solve the model analytically and one only has numerical results to support the picture outlined above.

As explained in Sec. I, there exists the bijection between ensembles of GCDT and a set of graphs with a finite number of faces such that the number of local maxima of the distance labeling in GCDT coincides with the number of faces in the set of graphs. Coupling matter to GCDT has been studied so far in the sense that the GCDT graphs considered are the graphs with a finite number of faces (including tree subgraphs), e.g., a multicritical one-matrix model for GCDT coupling to hard dimers [31] and the twomatrix model for coupling to Ising spins [27,28]. The present work is a continuation of the latter, the Ising case.

Apart from the study on coupling of matter to GCDT in the way originally defined, it is interesting to work on the matter coupling to the GCDT graphs which are the graphs with a finite number of faces, as we have studied in this paper. This is because we may have a chance to observe critical behaviors different from the known: in the case of the Ising model coupled to GCDT based on the two-matrix model, on the Boulatov-Kazakov critical curve (parametrized by $\theta$ ) that is characterized by infinitely many skeleton vertices and divergent fluctuations of Ising spins, one can recover the critical behavior of the $c=1 / 2$ conformal matter minimally coupled to $2 \mathrm{D}$ gravity while going down to the zero critical temperature with tuning $\theta \rightarrow 0$, one can reach the critical end point where the two kinds of critical curves, i.e., the Boulatov-Kazakov critical curve and the critical curve for dominant trees, meet, at which we have had a possibility to obtain a new critical behavior. In the previous paper [28], it has been shown that the continuum limit around the zero critical temperature can be taken if one scales $\theta$ to be of order $\varepsilon^{3}$. In this paper, we have tried to elucidate the physics around this zero-temperature critical point quantitatively to compute divergent behaviors of the number of vertices and a critical exponent.

We have shown that the continuum limit with the scaling $\theta \sim \varepsilon^{3}$ proposed by [28] leads to the fact that the number of skeleton vertices remains finite even in the continuum limit, meaning that it is the tree structure that determines the critical behavior, i.e., what we have found is the critical behavior identical to that of the Ising model on a 1D lattice chain or branched polymers, which is not satisfactory because this criticality is already known.

One of interesting findings in the present work is the existence of the scaling $\theta \sim \varepsilon^{a}$ where $0<a<3$ even when 
coupling to Ising spins. With this $a$-dependent scaling, the number of skeleton vertices as well as the number of vertices in the dressed propagators diverges as shown by Eq. (4.22); we then have found that one can obtain a nontrivial scaling of the magnetization with the size of the graph, namely, Eq. (4.28). This is indeed a new type of critical behavior in between the Boulatov-Kazakov criticality $(a=0)$ and the criticality of the 1D spin chain $(a=3)$.

However, the result is not entirely satisfactory since it essentially reflects the standard Ising spin behavior on sublattices consisting of planar $\phi^{3}$ skeleton graphs. The main problem seems to be the character of the ensembles of interpolating graphs. They are simply too inhomogeneous, since the Ising spins are noncritical on the tree subgraphs, except at strictly zero temperature, where they are trivially critical, like the 1D spin chain.

It would be interesting to find better homogeneous ensembles of graphs, i.e., ensembles which have only one well-defined fractal dimension, and which interpolate between the DT and the GCDT ensembles. On these ensembles, one might very well find new scaling behavior of the Ising model.

\section{ACKNOWLEDGMENTS}

We would like to thank Timothy Budd, Bergfinnur Durhuus, Masafumi Fukuma, Luca Lionni, Tadakatsu Sakai, Masaki Shigemori, and Hidehiko Shimada for fruitful discussions and encouragements. Y.S. visited the Niels Bohr Institute, Denmark, the Radboud University, the Netherlands, and the Shing-Tung Yau Center of Southeast University, China where part of this work was done. He would like to thank all the members there for the kind hospitality. J. A. acknowledges the support from the Danish Research Council, via the grant "Quantum Geometry," Grant No. 7014-00066B. The work of Y. S. was supported by Building of Consortia for the Development of Human Resources in Science and Technology and by JSPS KAKENHI Grant No. 19K14705.
[1] V. A. Kazakov, Ising model on a dynamical planar random lattice: Exact solution, Phys. Lett. A 119, 140 (1986).

[2] D. V. Boulatov and V. A. Kazakov, The Ising model on random planar lattice: The structure of phase transition and the exact critical exponents, Phys. Lett. B 186, 379 (1987).

[3] J. Ambjørn, B. Durhuus, and J. Frohlich, Diseases of triangulated random surface models, and possible cures, Nucl. Phys. B257, 433 (1985).

[4] J. Ambjørn, B. Durhuus, J. Frohlich, and P. Orland, The appearance of critical dimensions in regulated string theories, Nucl. Phys. B270, 457 (1986).

[5] F. David, Planar diagrams, two-dimensional lattice gravity and surface models, Nucl. Phys. B257, 45 (1985).

[6] A. Billoire and F. David, Microcanonical simulations of randomly triangulated planar random surfaces, Phys. Lett. 168B, 279 (1986).

[7] V. A. Kazakov, A. A. Migdal, and I. K. Kostov, Critical properties of randomly triangulated planar random surfaces, Phys. Lett. 157B, 295 (1985).

[8] D. V. Boulatov, V. A. Kazakov, I. K. Kostov, and A. A. Migdal, Analytical and numerical study of the model of dynamically triangulated random surfaces, Nucl. Phys. B275, 641 (1986).

[9] J. Ambjørn, B. Durhuus, and T. Jonsson, Quantum Geometry: A Statistical Field Theory Approach, Cambridge Monographs on Mathematical Physics (Cambridge University Press, Cambridge, United Kingdom, 1997).

[10] L. Onsager, Crystal statistics. 1. A two-dimensional model with an order disorder transition, Phys. Rev. 65, 117 (1944).
[11] V. G. Knizhnik, A. M. Polyakov, and A. B. Zamolodchikov, Fractal structure of 2D quantum gravity, Mod. Phys. Lett. A 03, 819 (1988).

[12] J. Ambjørn and R. Loll, Nonperturbative Lorentzian quantum gravity, causality and topology change, Nucl. Phys. B536, 407 (1998).

[13] J. Ambjørn, J. Jurkiewicz, and R. Loll, A Nonperturbative Lorentzian Path Integral for Gravity, Phys. Rev. Lett. 85, 924 (2000).

[14] J. Ambjørn, J. Jurkiewicz, and R. Loll, Dynamically triangulating Lorentzian quantum gravity, Nucl. Phys. B610, 347 (2001).

[15] J. Ambjørn, A. Goerlich, J. Jurkiewicz, and R. Loll, Nonperturbative quantum gravity, Phys. Rep. 519, 127 (2012).

[16] R. Loll, Quantum gravity from causal dynamical triangulations: A review, Classical Quantum Gravity 37, 013002 (2020).

[17] J. Ambjørn, K. N. Anagnostopoulos, and R. Loll, A new perspective on matter coupling in 2-D quantum gravity, Phys. Rev. D 60, 104035 (1999).

[18] J. Ambjørn, K. N. Anagnostopoulos, and R. Loll, Crossing the $c=1$ barrier in 2-D Lorentzian quantum gravity, Phys. Rev. D 61, 044010 (2000).

[19] J. Ambjørn, A. T. Goerlich, J. Jurkiewicz, and H.-G. Zhang, Pseudo-topological transitions in 2D gravity models coupled to massless scalar fields, Nucl. Phys. B863, 421 (2012); A $c=1$ phase transition in two-dimensional CDT/ Horava? Lifshitz gravity? Phys. Lett. B 743, 435 (2015); The microscopic structure of 2D CDT coupled to matter, Phys. Lett. B 746, 359 (2015). 
[20] J. Ambjørn, B. Durhuus, and T. Jonsson, A solvable 2-d gravity model with gamma $>0$, Mod. Phys. Lett. A 09, 1221 (1994).

[21] J. Ambjørn, R. Loll, W. Westra, and S. Zohren, Putting a cap on causality violations in CDT, J. High Energy Phys. 12 (2007) 017.

[22] J. Ambjørn, R. Loll, Y. Watabiki, W. Westra, and S. Zohren, A string field theory based on causal dynamical triangulations, J. High Energy Phys. 05 (2008) 032.

[23] J. Ambjørn, R. Loll, Y. Watabiki, W. Westra, and S. Zohren, A new continuum limit of matrix models, Phys. Lett. B 670, 224 (2008).

[24] J. Ambjørn and T. G. Budd, Trees and spatial topology change in CDT, J. Phys. A 46, 315201 (2013).

[25] J. Ambjørn, R. Loll, Y. Watabiki, W. Westra, and S. Zohren, A matrix model for 2D quantum gravity defined by causal dynamical triangulations, Phys. Lett. B 665, 252 (2008).

[26] J. Ambjørn, T. Budd, and Y. Watabiki, Scale-dependent Hausdorff dimensions in 2d gravity, Phys. Lett. B 736, 339 (2014).
[27] H. Fuji, Y. Sato, and Y. Watabiki, Causal dynamical triangulation with extended interactions in $1+1$ dimensions, Phys. Lett. B 704, 582 (2011).

[28] Y. Sato and T. Tanaka, Criticality at absolute zero from Ising model on two-dimensional dynamical triangulations, Phys. Rev. D 98, 026026 (2018).

[29] J. Ambjørn, B. Durhuus, T. Jonsson, and G. Thorleifsson, Matter fields with $c>1$ coupled to 2-d gravity, Nucl. Phys. B398, 568 (1993).

[30] H. Kawai, N. Kawamoto, T. Mogami, and Y. Watabiki, Transfer matrix formalism for two-dimensional quantum gravity and fractal structures of space-time, Phys. Lett. B 306, 19 (1993); J. Ambjørn and Y. Watabiki, Scaling in quantum gravity, Nucl. Phys. B445, 129 (1995); J. Ambjørn, J. Jurkiewicz, and Y. Watabiki, On the fractal structure of two-dimensional quantum gravity, Nucl. Phys. B454, 313 (1995).

[31] J. Ambjorn, L. Glaser, A. Gorlich, and Y. Sato, New multicritical matrix models and multicritical 2d CDT, Phys. Lett. B 712, 109 (2012). 